GENEVIEVE MICHON, HUBERT DE FORESTA ET PATRICE LEVANG

\author{
Les populations javanaises qui exploitent les forêts pour en extraire la résine \\ ont-elles mis au point des systèmes d'agroforesterie susceptibles de servir \\ de "modèles" dans une perspective de développement durable?
}

L'Indonésie représente, juste après l'Amazonie, la deuxième réserve de forêts tropicales dans le monde. Dans tout l'archipel, la forêt joue un rôle essentiel non seulement comme source de biens commercialisables mais aussi pour l'ensemble des besoins matériels et culturels des populations locales. Malgré un développement agricole important, plusieurs millions de personnes habitent encore dans des zones forestières et dépendent plus ou moins étroitement des ressources forestières. L'État indonésien, lui, tire une grande partie de ses richesses de l'exploitation des forêts naturelles. En 1992, les bois de sciage et les contreplaqués représentaient à eux seuls $12,4 \%$ des exportations nationales.

L'histoire des forêts en Indonésie se confond avec celle d'un long et laborieux processus d'appropriation par l'État aux dépens des communautés locales. Les modes de gestion dominants des ressources forestières sont aujourd'hui marqués par une vision réductionniste omniprésente, qu'il s'agisse de la biodiversité, des fonctions économiques de la forêt, ou du partage des bénéfices qui en sont tirés. La contribution importante de l'industrie du bois à l'économie nationale ne suffit plus à masquer les dégâts de plus en plus visibles de la gestion des forêts sur les plans écologique, économique ou social. La réduction du couvert forestier, le gaspillage et la disparition des ressources, la mise à l'écart de toute une frange de la population rurale commencent à faire l'objet d'un débat national. Face à ces problèmes, l'État opte plus volontiers pour la mise en réserve des forêts naturelles, pour la conversion en plantations industrielles ou en zone de coloni-

\section{RÉSUMÉ : Stratégies agroforestières paysannes et développement durable : les agroforêts à damar de Sumatra}

En Indonésie, les conflits entre l'État et les populations locales pour l'usage et le contrôle des ressources conduisent depuis une dizaine d'années à une dilapidation accélérée des ressources forestières. Cette dilapidation s'accompagne d'une dégradation rapide du milieu et la question du développement durable des zones forestières est largement au cœur des débats nationaux. II existe pourtant des exemples d'évolution positive de systemes de gestion paysans des ressources forestières, en particulier dans le domaine agroforestier.

Entre plantation et forêt, les "agroforêts" indonésiennes représentent une stratégie originale d'appropriation et d'usage de ressources naturelles par les communautés paysannes, combi- nant logique agricole et reconstruction forestière.

A travers I'histoire et l'analyse d'une agroforêt de Sumatra, nous tenterons d'établir quelle peut être la contribution de ce concept d'agroforêt au débat sur la dynamique et l'usage des ressources renouvelables dans le cadre du développement durable. Mettant l'accent sur les mécanismes biologiques et les stratégies sociales qui ont permis, plus que la conservation d'une ressource forestière particulière, la restauration de la "ressource forêt" dans son ensemble, nous réfléchirons à la viabilité de cette "stratégie agroforestière" dans un contexte particulièrement défavorable au contrôle des ressources forestières par les paysans. sation agricole que pour des modèles de gestion alternatifs. Les propositions de mise en valeur des produits forestiers mineurs, d'utilisation diversifiée des forêts et de gestion par les populations locales, même si elles recueillent l'assentiment des pouvoirs publics, ne sont pas encore suivies d'effets.

Au niveau national, le débat sur les modes d'utilisation et les conditions de dégradation des ressources en zone forestière reste encore dominé par une perception négative de l'action des paysans de la part des décideurs. Les exemples performants de gestion paysanne des ressources sont sous-estimés ou mal compris. Depuis longtemps coincés entre un cadre institutionnel limitant fortement l'accès aux forêts naturelles et une réalité économique incitant à l'utilisation intensive de leurs ressources, les paysans des
1. Cette étude a été réalisée dans le cadre du programme de I'UR $3 \mathrm{H}$ (département milieux et activité agricole, ORSTOM). Le programme, réalisé en coopération avec le Biotrop de Bogor (1990-1994) et avec I'ICRAF (depuis 1994), a bénéficiè d'un financement complémentaire du ministère de l'Environnement français (programme SOFT de 1990 à 1993) et de l'action incitative DURR (Dynamique et usages des ressources renouvelables). Les auteurs expriment leurs plus sincères remerciements à Mme Claudine Friedberg pour les nombreuses améliorations apportées au manuscrit. 
marges forestières de l'archipel ont pourtant su mettre au point des systèmes tout à fait remarquables. En adaptant d'anciens modes de gestion de la forêt à une logique d'agriculture commerciale, ils introduisent au sein des terroirs des structures typiquement forestières : les agroforêts.

Nous essayerons d'expliquer dans un premier temps comment les différences de perception qui existent entre pouvoir central et populations des îles forestières au sujet des forêts et de leurs ressources ont pu conduire à la situation actuelle de pillage des ressources forestières. Puis, à travers l'histoire et l'analyse d'une agroforêt du sud de Sumatra, nous tenterons d'illustrer ce que ce concept d'agroforêt peut apporter au débat sur la dynamique et l'usage des ressources renouvelables dans le cadre du développement durable. Nous défendrons la thèse que le développement d'agroforêts constitue une stratégie originale et efficace sur les plans économique, écologique et socioculturel, d'appropriation ou, plus souvent, de réappropriation des ressources forestières par des communautés paysannes.

Nous partirons de l'exemple de l'évolution de la gestion d'une ressource forestière - la résine damar - depuis d'anciens systèmes extractivistes jusqu'à la mise en culture au sein d'un système agroforestier original. Nous nous attacherons d'abord à démontrer comment l'intégration des processus biologiques forestiers dans le système agricole, combinée à une gestion économique diversifiée, a permis, outre la conservation de la ressource damar en particulier, la restauration de la "ressource forêt" dans son ensemble. Nous verrons aussi comment la création et l'extension des agroforêts ont modifié les systèmes de représentation, institutionnels et sociaux, qui sous-tendent la gestion des ressources forestières et comment cette modification a renforcé le processus d'appropriation des ressources forestières par les paysans. Enfin, nous analyserons comment la gestion globale des ressources forestières pourrait évoluer dans un sens totalement original en s'intégrant davantage à l'agriculture. En conclusion, nous réfléchirons à la viabilité d'une stratégie agroforestière de gestion des terres forestières dans un contexte idéologique et institutionnel particulièrement défavorable au renouvellement de ressources naturelles dans les systèmes de production paysans.

\section{PAYSANS DES ÎLES PÉRIPHÉRIQUES ET POUVOIR CENTRAL : UN CONFLIT ANCIEN}

La gestion des terres et des ressources forestières en Indonésie est marquée, tout au long de son histoire, par l'opposition quasi constante entre communautés locales et pouvoir central.

\section{La forêt : centre du monde...}

Pour les populations d'essarteurs de Sumatra et de Kalimantan, la plupart des mythes d'origine de la société et d'accès aux connaissances impliquent des mariages entre humains et génies de la forêt. La forêt, milieu de vie et d'activités essentiel, n'est pas ressentie comme extérieure à la sphère agricole. Elle est perçue comme source de produits individualisés obtenus à partir d'espèces particulières, mais aussi dans sa globalité en tant qu'écosystème comme "ressource forêt", notion liée à une gestion intégrant le long terme. Ressentie comme un monde à " utilisation multiple ", la forêt apparaît comme une somme de faciès dont chacun peut être défini par une gamme d'activités possibles, ou par une somme de biens extractibles.

On peut discerner deux pôles d'utilisation de la forêt. L'un concerne des ressources liées à la subsistance, l'autre celles utilisées pour les échanges commerciaux.

Parmi les premières, on retrouve des produits végétaux et animaux, alimentaires ou ayant une autre utilité. Permettant la reproductibilité de l'agriculture sur brûlis, la dynamique de régénération de la forêt peut également être considérée comme une ressource essentielle pour la subsistance des populations forestières. Cependant ces ressources perdent chaque jour de leur importance, d'une part, sous l'effet d'une meilleure intégration des villages forestiers à l'économie nationale qui amène aliments et produits manufacturés sur les marchés et, d'autre part, sous une pression démographique croissante qui se traduit par la réduction de la durée des jachères arborées et par la perturbation de la dynamique forestière.

Les ressources commerciales sont multiples. Leur importance respective a considérablement évolué au cours de l'histoire, en fonction surtout des fluctuations du marché. Historiquement, la forêt indonésienne est d'abord prisée pour ses produits rares (encens, plumes, ivoire, corne de rhinocéros, épices). Puis pour ses résines et ses latex. Enfin pour ses rotins, et aujourd'hui pour son bois. L'importance des échanges fondés sur les produits forestiers n'a pas faibli. Les produits de la forêt, qui ont lié dès les premiers siècles de notre ère les populations forestières de Sumatra ou de Bornéo au reste du monde, sont encore aujourd'hui essentiels pour l'économie de la plupart des populations agricoles de ces régions. Ces ressources commerciales forestières sont l'objet d'un antagonisme fondamental entre les autochtones et l'État.

Le contrôle de l'accès aux ressources par les communautés locales met en jeu toute une panoplie de droits, allant d'un type de gestion très communautaire à la propriété privée des terres et des ressources. On y observe une dissociation fréquente entre les modes de contrôle ou d'accès à la terre et ceux qui concernent les ressources. Ces derniers sont en général adaptés à la nature des ressources, à la biologie et à l'écologie des espèces qui les produisent. Celles dont la régénération est incertaine ou lente sont de préférence sous le contrôle direct de la communauté. Dans les quelques cas d'appropriation privée, la communauté maintient un droit de regard qui vise essentiellement à assurer la reproductibilité ou la pérennité des ressources concernées. 


\section{...ou frontière du monde civilisé ?}

Pour l'État, la forêt est un enjeu géopolitique et économique. La vision officielle de la forêt dans l'Indonésie du XX' siècle est marquée à la fois par la place qu'elle occupe dans la culture javanaise, prisant plus les clairières défrichées que la sylve et plus l'agriculture permanente en rizières irriguées que les systèmes itinérants (Dove, 1985 ; Peluso, 1992 b) et par trois siècles de domination néerlandaise durant lesquels les forestiers coloniaux ont imposé une conception occidentale réellement réductrice de la forêt considérée sous le seul angle de la productivité. Aujourd'hui, la forêt apparaît comme une source de produits commercialisables mais également comme un frein au développement, parce que son territoire est considéré comme une réserve foncière qu'il faut défricher. De par son immensité et son exubérance, la forêt est encore perçue comme une ressource infinie et inépuisable. Le fait d'en toucher de très près les limites n'ébranle que peu cette vision.

Sous la domination néerlandaise ou sous 1'"ordre nouveau" mis en place en 1967 , le pouvoir central a toujours cherché à se rendre maitre de la forêt, de ses ressources et de ses populations. Actuellement, du point de vue juridique et institutionnel, l'utilisation et la gestion des forêts sont codifiées par la loi forestière de 1969. La première fonction de cette loi forestière consiste à fixer les limites et les fonctions du domaine forestier national.

Ainsi, $74 \%$ du territoire, considérés comme "terres forestières" et propriété légale de l'État, sont placés sous la tutelle du ministère des Forêts. Les droits coutumiers ne sont pas niés puisque la Constitution les reconnaît de manière explicite... tant qu'ils " n'interfèrent pas avec les intérêts supérieurs de la Nation ». La formulation reste suffisamment ambiguë pour permettre les interprétations les plus larges comme les plus étroites.

L'appropriation des forêts par l'État s'appuie aussi sur un contrôle de plus en plus total de l'utilisation et des utilisateurs du milieu. Depuis une vingtaine d'années, la dimension économique de la forêt se résume, pour l'État, à sa composante ligneuse, qu'il exploite en s'associant presque exclusivement à des entreprises privées.

\section{La dilapidation \\ du patrimoine commun}

Considérer la forêt tropicale comme une ressource renouvelable peut prêter à discussion. Toutefois, il est indéniable que les modes d'exploitation actuels condamnent irrémédiablement son renouvellement. De nombreuses études démontrent que la confrontation entre l'État, le marché, les sociétés indigènes et les compagnies privées se traduit par une dilapidation accélérée des ressources forestières (Durand, 1994). Tous les acteurs sociaux, des agents de contrôle aux populations locales, participent au gaspillage. L'exploitation du bois en concession interdit pratiquement aux populations locales l'accès aux autres ressources de l'écosystème pendant la durée du contrat. Les dégâts qu'entraîne cette exploitation souvent abusive mettent en difficulté les populations forestières. Les cas de dépossession des communautés autochtones se multiplient et la plupart des systèmes traditionnels de contrôle de l'ensemble des ressources forestières (Gillis, 1988) sont irrémédiablement condamnés. L'intrusion massive de collecteurs extérieurs provoque souvent une dérégulation des systèmes traditionnels entrainant une surexploitation anarchique de la part des autochtones (Siebert, 1989 ; Peluso, 1992 a).

De plus en plus, la forêt devient le domaine exclusif du court terme, chacun privilégiant son intérêt immédiat, quitte à compromettre son avenir. Le développement durable des terres forestières se heurte aujourd'hui autant à des problèmes techniques qu'à des difficultés sociales et culturelles. Les seules forêts contrôlées de façon durable par les communautés locales sont des "forêts" anthropisées, reconstituées au sein du terroir agricole. 


\section{LES RÉSINES DAMAR : DE LA CUEILLETTE À LA PLANTATION}

\section{Une histoire ancienne}

Les résines damar ${ }^{2}$ sont produites par une centaine d'espèces appartenant à la famille des Diptérocarpacées, famille ligneuse dominante dans les forêts de basse altitude en Indonésie. Les damar batu (littéralement damar pierre), exsudats spontanés formant de gros blocs solides récoltés au pied des troncs, se distinguent des damar mata kucing (damar œil-de-chat), résines claires de qualité supérieure récoltées par incision de l'écorce (figure 1). La collecte de résines sauvages constitue l'une des activités forestières les plus anciennes en Asie du Sud-Est. Produit d'échanges entre les Îles indomalaises dès le $\mathrm{III}^{\leftarrow}$ millénaire avant notre ère, certaines résines de Sumatra sont commercialisées jusqu'en Chine à partir du $1 I^{e}$ siècle de notre ère (Dunn, 1975). Les damar, mentionnés dans des écrits chinois du $\mathrm{x}^{\mathrm{e}}$ siècle, ne sont exportés vers l'Europe qu'après 1820 . Servant pour le calfatage des bateaux et la fabrication de torches, ils servaient également de base pour la confec. tion d'encens et de peintures. Avec le développement de la fabrication industrielle de peintures et de vernis, les damar sauvages connaissent un engouement sans précédent au milieu du siècle dernier. Entre 1850 et 1920 , la collecte commerciale de damar représente une activité essentielle pour de nombreuses populations de Sumatra et de Bornéo.

Au début du siècle, le sud de Sumatra et plus particulièrement la région de Krui (également appelée Pesisir) sur la côte ouest de la province de Lampung (voir carte) constitue l'une des principales régions exportatrices de damar mata kucing. Dans le Pesisir, l'essentiel de la production provient d'une seule espèce : Shorea javanica K \& V. Alors que la riziculture sur brûlis assure l'autosuffisance alimentaire des familles, la production de poivre (ou de café) et la collecte de damar leur permettent d'accéder à un revenu monétaire. À partir de la deuxième moitié du XIX siècle, la flambée des

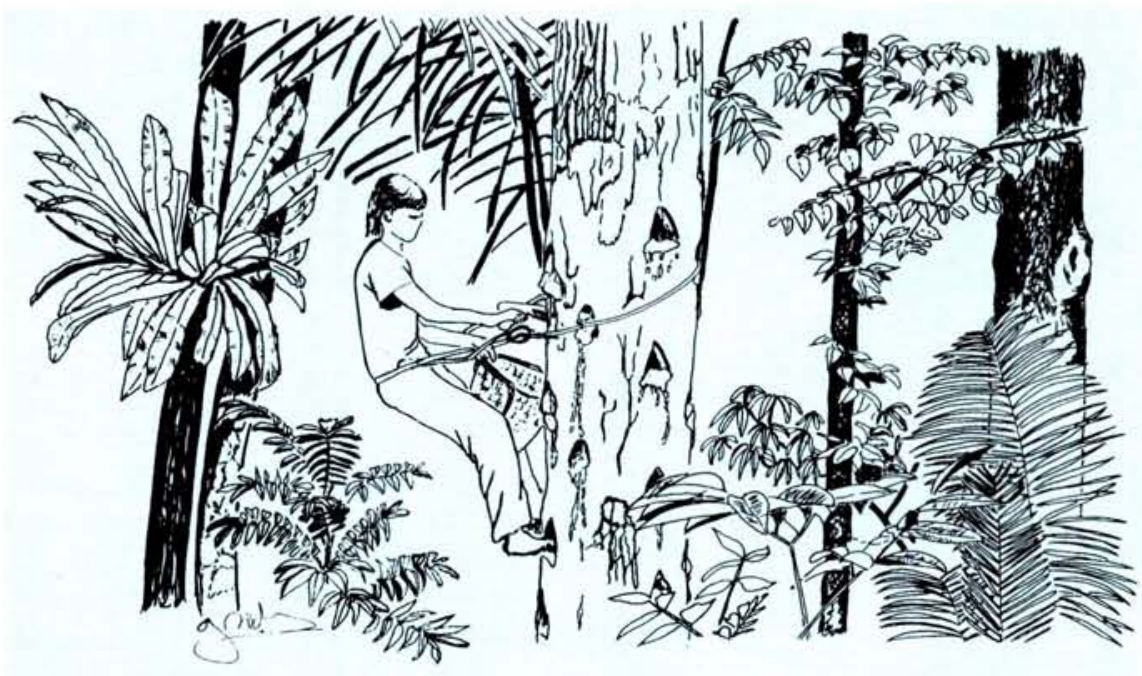

Figure 1-Récolte du damar mata kucing produit par Shorea javanica à Sumatra (dessin G. Michon).

prix du damar se traduit par une cueillette excessive. Saignés abusivement, les arbres meurent précocement. La raréfaction des individus reproducteurs compromet fortement la régénération naturelle, tandis que l'extension continue du territoire cultivé fait reculer la ressource forestière dans son ensemble. Vers 1920, dans tout le sud de Sumatra, le damar est devenu une ressource

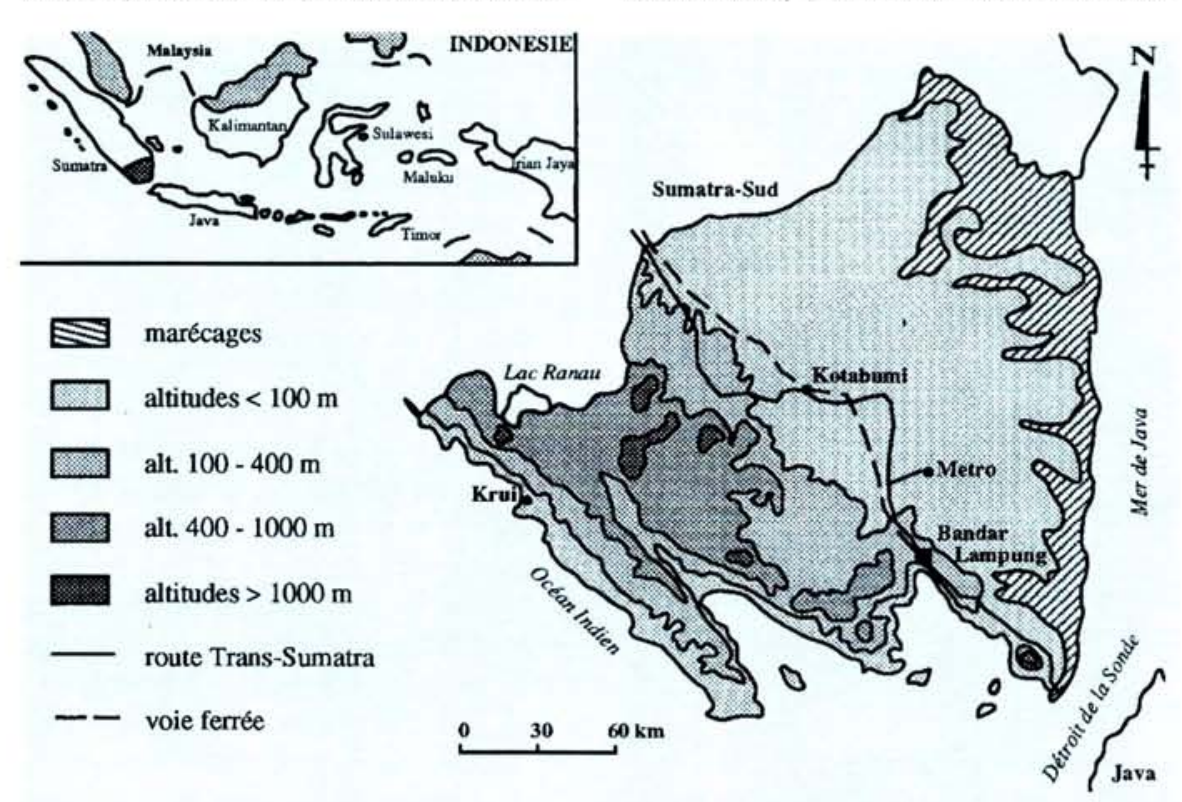

La province du Lampung (localisation de la zone d'étude). rare et se renouvelant difficilement. Aux problèmes biologiques s'ajoutent des problèmes sociaux. Jusque-là, le "découvreur" d'un arbre - en fait le premier à le saigner disposait d'un droit de saignée permanent et exclusif, reconnu par sa communauté territoriale et les communautés avoisinantes. Mais devant le soudain afflux de cueilleurs allochtones, le contrôle communautaire 
n'apparaît plus en mesure de défendre l'accès à la ressource individuelle. La crainte de voir des "étrangers" s'approprier indûment le fruit de leur travail incite les ayants droit à surexploiter leurs arbres, qui s'épuisent vite. Avec les vols, les conflits entre villages se multiplient. En 1935, un forestier néerlandais en visite dans la région n'hésite pas à conclure à la disparition des damar sauvages sur toute la côte (Rappard, 1937).

C'est également à cette époque que les plantations villageoises de damar commencent à se développer dans le Pesisir. La mise en culture de l'arbre est probablement plus ancienne. Selon divers témoignages, elle remonterait au dernier quart du $\mathrm{XIX}^{e}$ siècle. Par contre, l'introduction systématique du damar dans le système de culture n'intervient pas avant 1927. En 1935, Rappard compte déjà 70 hectares de jardins à damar en production. En 1994, la surface totale de jardins en production s'élève à plus de 10000 hectares, auxquels il convient d'ajouter quelques milliers d'hectares de jeunes plantations qui entreront en production dans les 15 ou 20 années à venir.

Aujourd'hui, plus de $80 \%$ de la résine damar produite en Indonésie provient des jardins du Pesisir. Sur les 70 villages de la région, 57 comptent plus de la moitié de leur population active impliquée dans la production de damar (Dupain, 1994).

Encore menacés de disparition au début du siècle, les damar, aujourd'hui innombrables, dominent le Pesisir de leurs cimes imposantes. Une cinquantaine d'années seulement auront suffi aux paysans de la région pour passer d'une disparition irréversible à une multiplication massive, ce qui représente une véritable performance en matière de dynamique forestière.

\section{Une véritable sylviculture}

Loin d'être parfaite, la maîtrise technique du damar dans le Pesisir constitue cependant une prouesse dans la mesure où les paysans ont concrétisé ce qui pour nombre de forestiers reste un rêve (Michon, 1985). À savoir, établir, maintenir et reproduire, à faible coût et sur de grandes surfaces, une plantation saine de Diptérocarpacées. Le Shorea javanica, à l'instar de nombreuses Diptérocarpacées, fructifie peu, régénère mal dans la nature, et n'entre en production qu'au bout d'une vingtaine d'années (à peu près une génération humaine). Plutôt que de s'appuyer sur une modification des caractères biologiques de l'arbre selon le processus "classique" de la domestication, la maitrise de la culture du damar s'est faite de façon tout à fait originale. C'est en effet grâce à la reconstitution d'un écosystème forestier selon les principes de base de la dynamique forestière, que la plante a pu s'établir, se maintenir et se reproduire comme dans son milieu d'origine (figure 2).

Les plants de damar sont introduits depuis les pépinières villageoises dans une parcelle défrichée pour la culture du riz pluvial, soit après un premier cycle de riz, soit, plus fréquemment, dans la jeune plantation de caféiers ou de poivriers qui fait généralement suite aux cultures de riz. L'association avec le caféier ou le poivrier, dont le cycle reste inférieur à une dizaine d'années, équivaut à une phase de colonisation contrôlée, qui permet aux jeunes damar d'accomplir dans les meilleures conditions les premiers stades de leur développement, sans avoir à trop souffrir de la concurrence avec la végétation pionnière naturelle. Lorsque les caféiers ou les poivriers cesseront d'être entretenus, les damar continueront à se développer au sein de la végétation secondaire. La structure de la plantation que les villageois appellent "jardin" se complexifiera avec les années, grâce à l'action combinée de l'homme qui introduit d'autres espèces utiles (fruitiers, palmiers, etc.) et des phénomènes naturels de diversification qui permettent le rétablissement des espèces forestières.

La phase "mûre" de la plantation de damar, qui commence à se stabiliser après $40-50$ ans, ressemble plus à une forêt qu'à une plantation classique. Elle comporte de nombreuses espèces utiles associées au
2. Damar est un terme indonésien designant à la fois la résine et l'arbre qui la produit. Il est ici utilisé de la même façon. 
a)
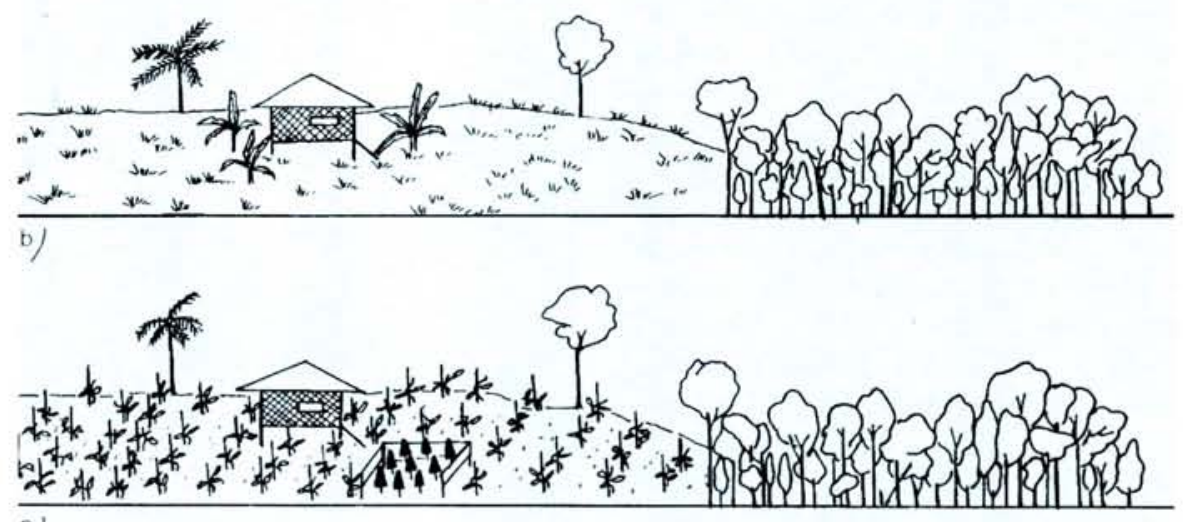

c)

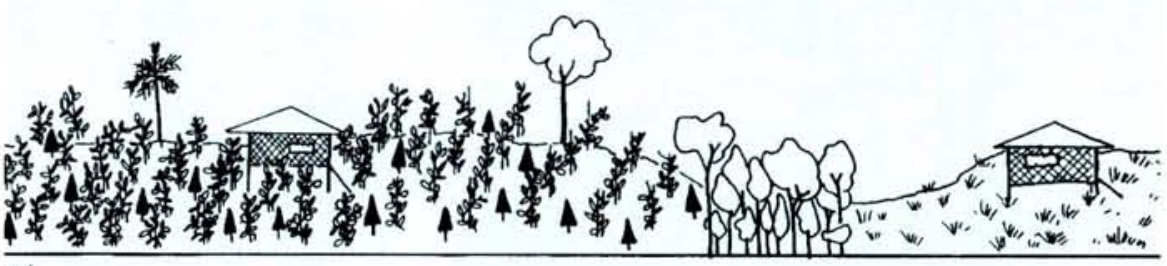

(5)
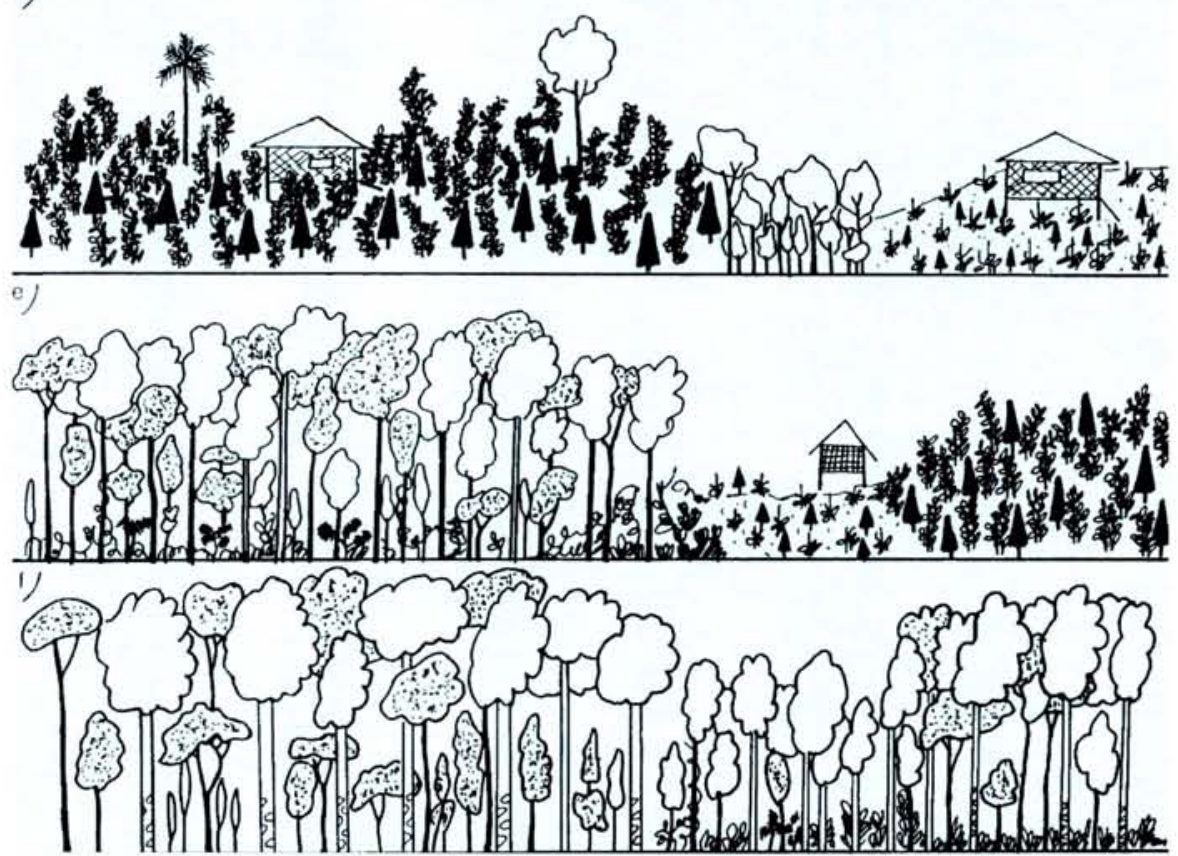

Figure 2 : Schèma d'établissement des agroforèts à damar : le ladang est ouvert sur d'anciennes jachères arborées, et planté en riz pluvial et en poivriers (a : année 1). Les damar, après un passage en pépinière pendant que les poivriers commencent à produire ( $\mathrm{b}$ : années 2 à 3), sont plantés au cours de la quatrième année (c), et bénéficient des soins apportés aux poivriers jusqu'à ce que ces derniers soient abandonnés ( $\mathrm{d}$ : années 8-10). Les damar se développent alors au sein de la végétation secondaire jusqu’ả leur entrée en production vers l'âge de 25 ans (e). La mosaïque de champs temporaires et de jachères arborées, caractéristique de l'agriculture sur brûlis, est maintenant remplacée par une couverture arborée permanente, l'agroforèt à damar (f). damar, et la végétation naturelle n'est pas systématiquement combattue. On n'observe plus de perturbation majeure équivalente à la phase d'installation car les vieux arbres sont remplacés au fur et à mesure des besoins. Le jardin à damar du Pesisir est en fait proche du modèle occidental des "futaies jardinées", forêts gérées de façon très extensive, dans lesquelles les arbres sont récoltés et régénérés individuellement. Une fois établie, la "futaie" de damar reste forêt, spécialisée mais en même temps diversifiée, sans retour ultérieur à une phase de régénération massive.

\section{Entre ager et sylva : l'agroforêt...}

Biologiquement parlant, les jardins à damar sont indéniablement proches d'un écosystème forestier naturel. Les nombreuses espèces associées au damar - qu'elles soient ligneuses ou herbacées, cultivées ou spontanées, utiles ou non - confèrent aux jardins une diversité et une hétérogénéité toutes forestières. On retrouve ici à la fois une communauté végétale et animale d'une grande richesse et un ensemble de processus reproductibles à long terme selon ses dynamiques propres (Michon, 1985). Nombre de fonctions écologiques, économiques et sociales des jardins restent, elles aussi, typiquement forestières.

Cependant, les jardins sont établis dans une optique d'agriculture commerciale au sein d'un terroir agricole autrefois conquis sur la forêt. Ils sont appropriés par des techniques et selon des règles coutumières liées à l'activité agricole. Ils font désormais partie d'un système de production agricole (Mary, 1986) où ils deviennent les compléments indispensables de la rizière (figure 3).

Ce schéma illustre en fait la façon dont se sont mises en place les agroforêts á damar autour des villages de la région de Krui jusque vers la fin des années quarante : depuis cette époque, les caféiers sont associés aux poivriers ou les rempla. cent en tant que culture intermédiaire ; les vieilles jachères ayant pour la plupart été converties, l'expansion des agroforéts s'est poursuivie aux dépens de la forêt primaire. 


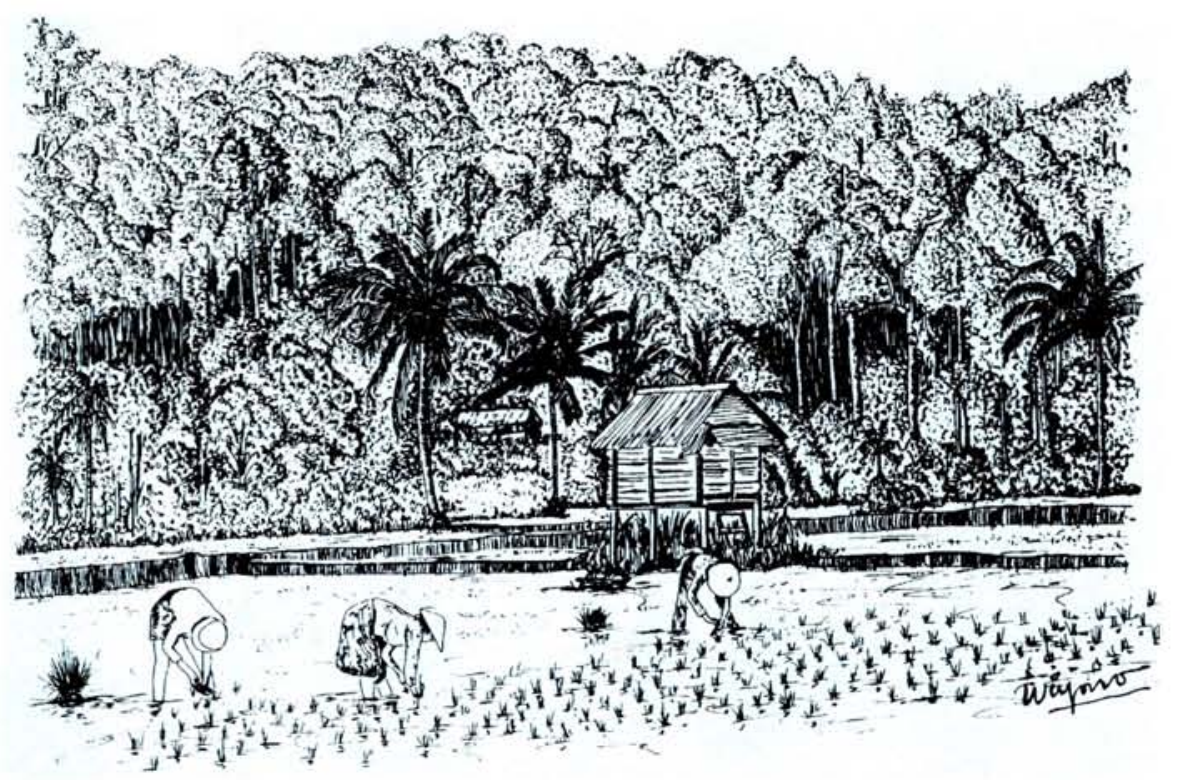

Figure 3 - L'agroforèt à damar est intégrée dans les terres agricoles au même titre que l'agriculture vivrière (dessin Wiyono).

Les jardins à damar occupent en fait cette vaste interface qui existe sous les tropiques entre forêt et domaine cultivé, mais qui reste difficilement concevable dans le modèle occidental moderne où la norme est d'opposer ager et sylva. Bien qu'ils ne puissent se définir comme une association permanente et intime entre cultures ligneuses et cultures herbacées, les jardins à damar se rattachent à la mouvance agroforestière, définie il y a une vingtaine d'années par les scientifiques. L'association rizière/jardins à damar peut être considérée comme un système agroforestier à partition horizontale de l'espace (Alexandre, 1989); leur mode d'établissement les rattache également aux systèmes agroforestiers séquentiels de type taungya (Nair, 1989); mais c'est surtout le jardin à l'état mature qui constitue un modèle agroforestier original, à savoir une association de diverses plantes pérennes, cultivées et spontanées, reproduisant une structure forestière complexe et intégrée dans un système de gestion agricole. De par leur faciès particulier et leur pérennité, les jardins à damar ont été définis comme des agroforêts (Michon, 1985 ; de Foresta et Michon, 1991).
AU-DELÀ DU DAMAR : LA RECONSTITUTION DE LA DIVERSITÉ FORESTIÈRE AU SEIN D'UN TERROIR AGRICOLE

L'originalité de l'histoire du damar dans le Pesisir ne tient pas uniquement à la prouesse technique liée à la mise en culture à grande échelle d'une espèce forestière. Elle provient surtout du fait qu'en introduisant une ressource forestière dans leur système de culture, les paysans ont réussi à reconstituer une véritable forêt (Michon, 1993).

Cette reconstitution forestière n'est pas a priori recherchée par les paysans. Elle peut apparaitre semi-intentionnelle, dans la mesure où les paysans introduisent de nombreuses plantes utiles parmi les damar. De plus, durant les années qui précèdent l'entrée en production, ils laissent s'installer des espèces spontanées. Ces deux facteurs de diversification confèrent très tôt au jeune jardin une allure forestière (figure 4). Mais la reconstitution de la biodiversité forestière apparait surtout $a$ posteriori. Elle est en fait la conséquence d'un mode de conduite particulier de l'agroforêt, qui tire parti des 


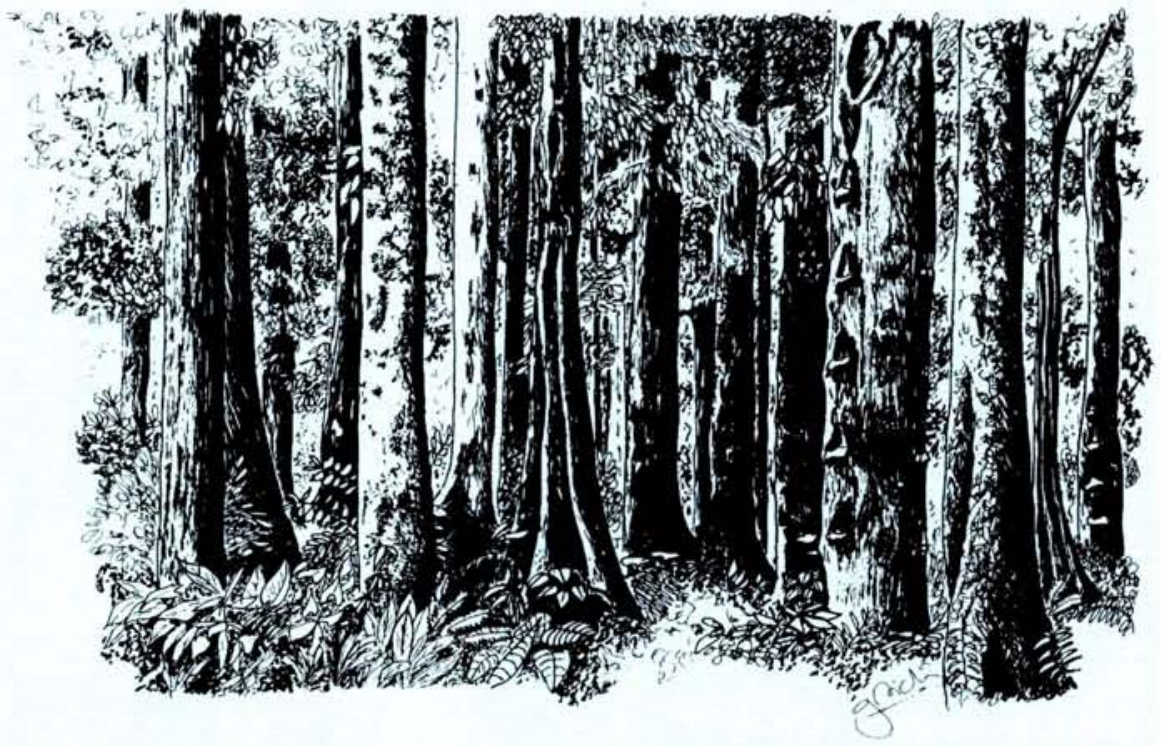

Figure 4 - Vue intérieure de l'agroforêt (dessin G. Michon).

dynamiques naturelles de production et de reproduction biologiques afin de réduire la contribution du facteur économique le plus rare : le travail. Une fois installée, l'agroforêt est en effet gérée selon une politique de soins minimum qui permet aux processus de diversification de se dérouler sans entraves, conformément aux règles de la dynamique forestière. Un jeune jardin à damar représente un ensemble de niches dans lesquelles des espèces végétales et animales issues des forêts avoisinantes peuvent se réinstaller et se reproduire. Le jeu des dispersions naturelles enrichit peu à peu la plantation. Dans une agroforêt mature, même si le damar reste dominant parmi les arbres, la composante végétale spontanée peut atteindre $50 \%$ du peuplement (Michon et de Foresta, 1994). Pour les herbacées, les lianes et les épiphytes, la composante spontanée est totalement dominante (figure 5). La plupart des mammifères forestiers de la région sont aussi présents dans l'agroforêt - plusieurs espèces rares telles que les gibbons et siamangs, la chèvre de Sumatra ou le rhinocéros de Sumatra, considérées comme menacées se rencontrent dans les agroforêts en tant qu'hôtes permanents ou transitoires (Sibuea et Herdimansyah, 1993) - et la richesse aviaire atteint environ $60 \%$ de celle des forêts voisines (Thiollay, 1994).

Très schématiquement, la reconstitution de la forêt se traduit par la conservation (ou la perte) de $50 \%$ de la biodiversité. Selon la sensibilité de l'observateur, le verre sera à moitié plein ou à moitié vide. Dans une pure optique de conservation, le vide l'emportera. Mais comparées aux plantations forestières modernes ne conservant guère qu'une espèce d'arbre et quelques oiseaux, les agroforêts à damar représentent une prouesse sans pareille dans le monde agricole et sylvicole.

Au-delà de la conservation in situ de ressources génétiques ou économiques, la restauration de la biodiversité a une autre signification : elle implique des processus biologiques déterminants pour la survie à long terme de l'agroforêt. Ce sont ces relations qui se nouent entre sol et plantes, entre plantes et animaux qui permettent le maintien à

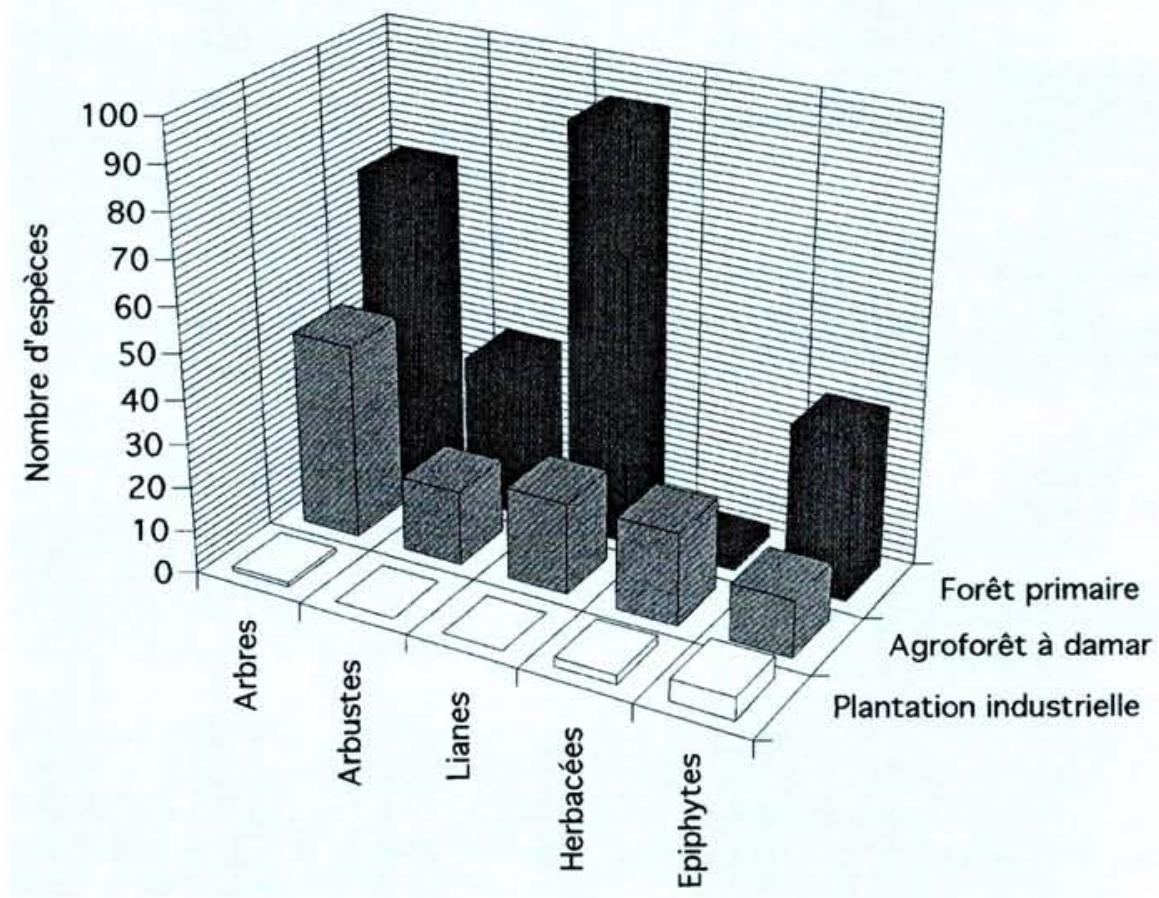

Figure 5 - Richesse floristique des grands groupes biologiques végétaux, dans une plantation industrielle, une agroforêt à damar et une forêt primaire (relevés = transects-lignes de $100 \mathrm{~m}$ de long; pour la plantation industrielle de damar, les données sont extrapolées à partir de celles d'une plantation d'hévéas). 


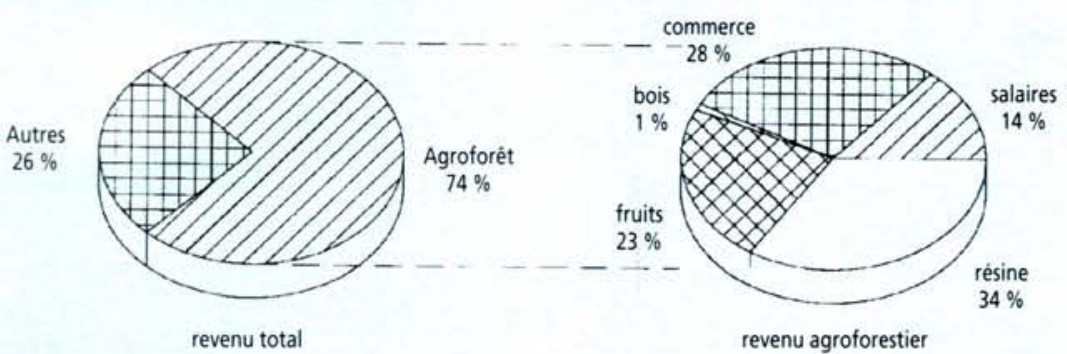

Figure 6 - Place des agroforêts à damar dans la formation du revenu monétaire des familles dans le village de Pahmungan.

peu de frais de l'agroforêt et garantissent la durabilité de son exploitation. Le libre déroulement des phénomènes de pollinisation, de dispersion, de décomposition, d'absorption, sont essentiels pour maintenir l'équilibre non seulement du fonctionnement global de l'agroforêt mais entre l'agroforêt, la forêt naturelle et les composantes du système agricole. Ces processus constituent de réelles "ressources fonctionnelles", qui n'ont aucune valeur économique directe, mais sans lesquelles les ressources économiques ne sauraient se reproduire.

\section{AU-DELÀ DE LA SPÉCIALISATION : UNE RÉAPPROPRIATION DE LA RESSOURCE FORÊT DANS L'ÉCONOMIE AGRICOLE}

La gestion économique de l'agroforêt est avant tout celle d'une plantation. Les jardins sont clairement établis dans une optique d'agriculture commerciale (Mary, 1986 ; Levang, 1989 ; Levang, 1992). Le damar, largement dominant dans l'agroforêt, fournit aujourd'hui la majeure partie des revenus monétaires des familles. La récente valorisation de certains fruits (durian, duku, petai) ${ }^{3}$ grâce à l'accès aux marchés urbains de Java, a encore accru la part provenant des agroforêts dans la constitution des revenus familiaux (figure 6). Un marché local du bois ${ }^{4}$ est en train de se mettre en place. Qui plus est, l'agroforêt, en tant que capital productif, peut faire office d'épargne de précaution. La mise en gage d'arbres ou de parcelles en production permet d'obtenir rapidement des prêts sans intérêt auprès des villageois fortunés. Tant que le prêt n'est pas remboursé, le prêteur dispose de l'usufruit de la parcelle selon un système classique de nantissement. L'emprunteur conserve la propriété de son bien sans limitation de durée. Fréquemment, il continue de saigner ses arbres, mais cette fois-ci sur la base d'un contrat de métayage au profit du prêteur.

En multipliant une ressource commercialisable, les jardins à damar ont permis de substituer aux anciennes stratégies de subsistance une stratégie commerciale destinée à pallier les défaillances prévisibles du système traditionnel de riziculture sur brûlis. En effet, comme dans d'autres régions, la croissance démographique et la réduction concomitante du couvert forestier menaçaient à terme la viabilité du système de production agricole dans son ensemble. Il s'agit là d'un processus d'intensification de l'agriculture en zone forestière que l'on retrouve ailleurs en Indonésie. Mais très souvent, l'intensification s'accompagne d'une spécialisation, aussi bien économique que biologique. Dans le Pesisir, il s'agit plutôt d'une diversification des structures productives commerciales, autrefois dominées par le poivre ou le café, aujourd'hui combinant damar, fruits, café, poivre et bois.

Bien que participant d'une logique de plantation commerciale, grâce à la reconstitution d'un niveau élevé de biodiversité permettant de reproduire la ressource forêt dans son ensemble, l'agroforêt a pu reprendre le rôle traditionnellement dévolu aux forêts naturelles, celui d'un espace à utilisation multiple, ouvert pour la cueillette et pour la
3. Respectivement Durio zibethinus Murr. Lansium domesticum Corr. et Parkia speciosa Hassk.

4. Essentiellement bois de coffrage et de construction. 
collecte commerciale et dont l'utilisation réelle est modulée par les besoins immé. diats.

L'agroforêt représente en effet une superposition d'espaces économiques souvent disjoints dans les systèmes de production agricole, superposition que l'on retrouve en partie dans l'utilisation traditionnelle des forêts naturelles. Elle est à la fois une source de revenu monétaire, un espace de production d'aliments et de matériaux et constitue une réserve mobilisable en cas de crise. L'agroforêt a permis le maintien de la chasse et de la pêche ou de certaines activités extractivistes liées à l'écosystème naturel comme la collecte de rotins. Aujourd'hui, les ressources sauvages - fruits, légumes, épices, matériaux de construction, combustibles, plantes médicinales - sont plus ou moins intensément utilisées pour la consommation immédiate, et l'on n'y recourt en général que dans les moments difficiles. Mais certaines d'entre elles représentent des ressources potentielles d'une grande valeur. Le bois, les rotins, certaines plantes médicinales et insecticides pourraient en effet, dans une optique de diversification des revenus agroforestiers, être commercialisés à grande échelle et devenir alors des spéculations agroforestières majeures (Mary, 1986; Levang, 1992 ; de Foresta et Michon, 1992).

Les fonctions sociales de l'agroforêt combinent elles aussi celles d'un espace agricole et celles d'une ressource forestière. Les ressources autres que le damar donnent lieu, comme dans une forêt, à des dons et à des échanges, importants à la fois pour maintenir une cohésion familiale entre membres distants d'un même lignage et pour redistribuer les richesses entre les membres d'une communauté villageoise fortement hiérarchisée. Mais, conformément à une vision agricole du monde, l'agroforêt représente également un patrimoine. Elle est le résultat d'une stratégie de création de propriété par défrichement de la forêt et le fruit d'un travail qui profitera surtout aux générations futures : l'agroforêt constitue un bien lignager inaliénable. Dans le contexte social et institutionnel très particulier du
Pesisir, cette notion de patrimoine apparaît essentielle. Elle permet à l'agroforêt de devenir l'assise foncière et productive d'un lignage. Enfin, elle constitue la base d'un système $\mathrm{d}$ '"assurance vieillesse" permettant aux enfants, une fois adultes, de prendre en charge leurs parents (Mary, 1986).

\section{ÉLABORATION DE NOUVEAUX SYSTÈMES DE PERCEPTION ET DE CONTRÔLE}

L'appropriation de l'ancienne ressource forestière par intégration au terroir agricole a entraîné de profonds bouleversements au niveau des systèmes de représentation et de contrôle. Mais, alors que la raréfaction de ressources importantes dans l'écosystème naturel entraine le plus souvent une dérégulation profonde des bases socioculturelles et institutionnelles de leur gestion, la conversion agroforestière dans le Pesisir a débouché sur leur réorganisation.

\section{De la forêt à l'agroforêt: une mutation essentielle}

Les systèmes anciens de perception et de représentation de la forêt et de ses ressources sont aujourd'hui oblitérés, du moins dans les villages arrivés à la limite de leur expansion territoriale. Disparaissant de l'environnement immédiat des villages, la forêt a perdu de son importance dans l'imaginaire des paysans. Parmi les raisons habituellement avancées pour expliquer cette mutation, le passage à un "islam adulte" figure en bonne place. L'islam n'a-t-il pas montré l'erreur de ces croyances anciennes, dans lesquelles les esprits et la magie tenaient une place prédominante? L'idéologie officielle en Indonésie soutient les grandes religions monothéistes dans leur lutte contre l'animisme. La forêt d'autrefois, domaine des esprits et source de richesses, était autant un monde imaginaire qu'un milieu de vie. La forêt d'aujourd'hui n'est ni mythique ni mystique, elle n'est plus que le domaine des administrateurs forestiers... et accessoirement celui des ennuis pour qui tente d'y pénétrer trop ostensiblement.
L'agroforêt n'a remplacé la forêt que dans le paysage, pas dans les systèmes de représentation. Elle reste avant tout un lieu cultivé, et les villageois utilisent en indonésien le mot kebun, qui signifie "jardin", "plantation", pour la désigner. Cependant dans le cas des agroforêts, la différence entre plantes cultivées et plantes spontanées reste floue. Les plantes commerciales, plantées ou non, ne bénéficient pas toutes du statut de "culture". Seule l'espèce dominante est réellement considérée comme telle. Il existe bien une distinction entre les espèces "plantées" et celles "poussant spontanément" mais de nombreuses espèces utiles entrant dans la catégorie "plantées" résultent souvent de germinations naturelles et non de plantation. L'appellation "plantée" indique plutôt que la plante en question peut être plantée ou transplantée. A contrario, les plantes "spontanées" correspondent à des espèces sauvages, dispersées depuis les forêts avoisinantes par le vent ou les animaux, existant de façon accidentelle dans un jardin, qui peuvent ou non être utilisées et protégées, mais surtout que personne n'a jamais songé à planter.

Dans la langue vernaculaire du Pesisir, les jardins proches du village sont appelés repong, terme qui désignait autrefois les jardins fruitiers privatifs détenus par les familles les plus anciennement établies. Les jardins plus éloignés sont appelés darak, terme générique qui désigne, à l'instar du ladang indonésien ou de l'essart français, toute parcelle défrichée en forêt par abattis et par brûlis. Le terme s'utilisera d'ailleurs indifféremment pour désigner un champ de riz pluvial, une caféière ou un jardin de damar. L'agroforêt ne sera assimilée à une forêt que dans le cadre d'activités bien spécifiques et pour la plupart liées à la forêt naturelle. Dans ce cas, l'appellation de "forêt" fait référence non à un jardin bien particulier, mais à l'espace agroforestier dans son ensemble. Ainsi, la "forêt" où l'on va chasser le cerf ou cueillir des rotins désigne aujourd'hui cet espace indifférencié couvert par l'agroforêt, les jachères et les reliques 
forestières qui s'étend entre les villages et la forêt interdite.

En permettant le maintien d'un mode de vie qui reste en continuité avec une tradition forestière dont elle est directement issue, l'agroforêt représente le dernier témoin d'une ancienne alliance. Toutes les relations entre l'homme et les ressources forestières passent aujourd'hui par elle. Le fait qu'elle ne soit pourtant pas assimilée par ses détenteurs à un faciès de forêt naturelle est significatif d'une évolution essentielle dans la perception de ces ressources. Les paysans, qui semblent avoir renoncé à toute prétention sur les forêts naturelles, domaine désormais exclusif de l'État, ont reconstruit, au sein d'un terroir agricole qu'ils pensent mieux contrôler, un espace privilégié dans lequel la ressource forestière est protégée.

\section{De la crise des communaux à la redéfinition des droits privés}

Institutionnellement, l'appropriation des ressources forestières via l'agroforêt s'accompagne de l'émergence de nouvelles valeurs : la propriété foncière et la privatisation des droits. L'extension des plantations de damar a entrainé une réorganisation totale des modes traditionnels de contrôle des terres et d'accès aux ressources naturelles. Selon le droit coutumier ancien, les terres et les ressources forestières étaient gérées en propriété commune par les villageois. Seules les terres aménagées en rizières irriguées et certaines terres situées immédiatement derrière le village et occupées par les jardins fruitiers repong donnaient lieu à une appropriation privée définitive et irrévocable, et seules les familles fondatrices des villages avaient accès à cette propriété privée de la terre. Le lignage, défini à la fois par une ascendance commune et par l'héritage foncier qui le fait vivre, ne se concevait alors que rattaché à la rizière. Les familles sans patrimoine foncier reconnu - branches cadettes et immigrants récents - vivaient essentiellement sur le domaine forestier réservé à l'agriculture itinérante.
Des droits individuels non transmissibles pouvaient être accordés pour certaines ressources forestières. Ainsi, pour les damar sauvages, la première saignée constituait le signe reconnu de l'appropriation. L'accès individuel à la terre était obtenu par le défrichement et la mise en culture, mais il s'agissait là d'un droit d'usage, la terre restant le bien de la communauté. Ces droits d'usage étaient en fait tacitement maintenus bien après l'abandon des cultures. La terre ainsi attribuée restait dans la famille du premier défricheur. Toutefois, le droit coutumier interdisait strictement de planter des arbres sur ces terres forestières.

L'introduction du damar sur les terres cultivées n'a pu être possible qu'après modification du droit coutumier (Levang, 1992). En autorisant l'implantation d'espèces pérennes dans les ladang, le droit coutumier avalise l'appropriation individuelle de terres faisant partie jusque-là du patrimoine collectif. Les jachères pouvant être revendiquées par n'importe quel membre de la communauté, la sécurisation du foncier passe par la "création" de jardins, c'est-à-dire par la plantation de ligneux à long cycle de vie. En très peu de temps, l'espace indivis, autrefois géré par la communauté et occupé par la forêt communale et les cultures sur brûlis, cède la place à un parcellaire de jardins privatifs. Mais cette privatisation ne s'est soldée ni par une individualisation totale de la gestion de l'agroforêt, ni par un affaiblissement du système social traditionnel. Elle a surtout permis aux familles autrefois sans patrimoine de se faire reconnaitre comme lignages. Leurs conditions d'existence et de continuité sont désormais indissociables de la possession d'un patrimoine agroforestier.

La définition de la propriété privée dans le Pesisir ne répond pas à la définition du droit romain - usus, fructus, abusus. La liberté de décision du propriétaire, en ce qui concerne l'abusus essentiellement, fait l'objet de nombreuses restrictions dont le respect est assuré non pas par le droit coutumier, mais par un système de contrôle social 
dans lequel l'individu s'efface devant le lignage.

Les modalités de transfert des jardins sont calquées sur les anciennes règles de transmission patrilinéaire appliquées aux rizières irriguées et aux jardins fruitiers. Selon ces règles, les biens lignagers se transmettent de fils aîné en fils aîné. Les cadets, exclus de l'héritage, ne peuvent accéder à la propriété que par la création de plantations, ce qui pose de graves problèmes dans les villages où terroir et finage se confondent (Mary, 1986 ; Mary et Michon, 1987). Les règles de transmission diffèrent en fonction de l'origine des biens. Un bien nouvellement créé est géré et transmis selon le bon vouloir de son propriétaire. Il entre dans la catégorie hak milik penuh ou droit de propriété entier. Il n'en va pas de même pour la propriété héritée (hak waris), même si juridiquement le hak waris est assimilé à un droit de propriété privée. En effet, si l'héritier dispose de la jouissance exclusive et totale de son bien, il n'en est pas moins res. ponsable devant son lignage. Il lui est interdit de vendre une partie du bien, ou même de couper des arbres sans avoir obtenu l'aval de tous les descendants vivants du créateur du bien en question. En fait, plus qu'un propriétaire au sens occidental du terme, l'héritier est le dépositaire d'un patrimoine dont la gestion est soumise au contrôle lignager. Transmettre est aussi important que recevoir. "Mon héritage n'est pas ma propriété " résume la philosophie du système de propriété dans le Pesisir. Il s'agit là d'une barrière réelle contre la privatisation totale et l'individualisation.

Si le système de propriété dans le Pesisir n'est en aucun cas assimilable à un régime de propriété commune ou collective (McKay et Acheson, 1987 ; Berkes, 1989), il reste toutefois indissociable du fonctionnement global du système social. Recevoir une propriété en héritage comporte d'importantes contreparties sociales et économiques. La transmission d'une propriété se fait en général après la naissance du premier enfant mâle du fils aîné. Le nouvel héritier devient alors chef d'une famille élargie qui comprend ses parents, ses frères cadets et leurs enfants, ainsi que ses sœurs célibataires, qu'il a charge d'héberger et de nourrir.

L'appropriation individuelle ne concerne pas la totalité du domaine agroforestier. En effet, de nombreuses ressources restent en propriété commune ou en accès libre. Il n'y a guère que le damar qui soit réellement contrôlé. Récolter la résine sans l'autorisation du propriétaire de l'arbre est un vol auquel se risquent uniquement les adolescents en mal d'argent de poche. Par contre, de nombreuses autres ressources dites "plantées", tels les fruits, le bois de feu, la sève du palmier à sucre, ou les bambous, restent globalement à la disposition de la collectivité - selon les ressources : le groupe domestique, le lignage ou le village - pour la consommation immédiate. Le prélèvement de petites quantités est toujours autorisé sans accord préalable du propriétaire. Par contre, des ponctions plus importantes, surtout si elles sont destinées à être commercialisées, nécessitent toujours l'autorisation du propriétaire et supposent un partage de la récolte ou du revenu obtenu. Pour les ressources dites "spontanées", plus typiquement forestières, qu'elles soient commerciales ou de subsistance, les règles oscillent entre propriété collective très élargie et accès libre (Schlager et Ostrom, 1992). Ainsi, la règle coutumière autorise, sans restriction concernant l'origine du collecteur et sur toute la surface de l'agroforêt, non seulement la chasse, la cueillette de légumes sauvages ou le ramassage de plantes médicinales, mais aussi des activités nettement plus rémunératrices comme la collecte commerciale de rotins.

\section{LA RECONSTRUCTION AGROFORESTIÈRE : LES LEÇONS D'UN TRANSFERT}

La stratégie agroforestière du Pesisir représente un exemple original d'appropriation de ressources forestières. Cette stratégie d'appropriation ne permet pas de conserver l'intégrité de l'écosystème naturel : la forêt climacique est largement détruite et l'agroforêt contribue pour une grande part à cette destruction. Mais elle permet la restauration de la plupart des ressources de l'écosystème de départ au sein d'une struc. ture artificielle mais biologiquement forestière.

Elle peut être comprise comme un exemple de transfert des ressources forestières depuis l'écosystème naturel jusqu'à un écosystème sinon totalement "cultivé", du moins mieux maîtrisé que la forêt naturelle. Ce transfert ne concerne pas seulement les ressources, mais aussi les structures biologiques qui en garantissent la pérennité. Il s'est accompagné d'une mutation des systèmes sociaux et des représentations qui sous-tendent la gestion des ressources naturelles.

Au niveau économique, l'objectif premier des paysans était bien évidemment de développer une stratégie commerciale viable sur le long terme pour parer aux défaillances de l'ancien système extractiviste. Cette stratégie s'appuyait sur la conversion de la forêt naturelle. Mais la disparition de la forêt n'a pas ici entraîné, comme cela s'observe souvent ailleurs, de réductionnisme économique. Elle n'a pas clos de façon irréversible les potentialités présentes dans l'écosystème originel. Au contraire, et ceci est intimement lié à la reconstitution de la diversité biologique : en construisant une agroforêt, les paysans ont su préserver toute une gamme de choix économiques pour le futur, ce qui constitue l'une des conditions essentielles d'un développement durable.

Si la tradition communautaire semble en général plus à même que la propriété individuelle de garantir dans le long terme le maintien à l'identique des ressources naturelles, dans le contexte indonésien l'appropriation privée favorise certainement les investissements, que ce soit en termes de travail individuel ou de capital. Dès lors qu'il y a besoin non seulement de gérer mais aussi de créer, de planifier, de transmettre des biens et des structures productives, c'est-àdire de passer d'une logique extractiviste à 
une logique de production, le cadre de la propriété privée, plus sécurisant pour les individus ou pour leur groupe domestique, s'avère aussi plus efficace.

De plus, dans le contexte actuel ${ }^{5}$ d'une perception très négative des régimes de propriété commune, censés conduire inéluctablement à la dégradation des ressources, la revendication claire et nette d'un droit privé permet aux villageois d'espérer s'assurer d'un meilleur contrôle des ressources dans le long terme et la reconnaissance de ce droit par les pouvoirs publics.

Cependant, par rapport à une appropriation individuelle stricte, le maintien du contrôle de la communauté sur l'usage des biens fonciers et des ressources qu'ils supportent s'avère lui aussi essentiel dans une optique de long terme. Il permet entre autres de contenir les initiatives privées dans certaines limites, fixées par des intérêts communautaires jugés supérieurs aux considérations individuelles.

Si l'agroforêt s'est d'abord développée en réponse à l'épuisement d'une ressource, elle constitue maintenant une réponse à la fermeture juridique des dernières forêts disponibles. En détruisant la forêt, puis en reconstituant une structure qui lui emprunte ses formes et ses espèces mais acquiert un statut agricole, le paysan revendique ses droits à s'approprier les ressources forestières en dépit des politiques gouvernementales qui renient la légitimité de ces droits.

\section{CÔTÉ FORÊT ? CÔTÉ JARDIN ? LA STRATÉGIE AGROFORESTIÈRE REVISITÉE}

Les agroforêts à damar ne constituent pas un cas unique en Indonésie. On retrouve ce type de gestion des ressources naturelles un peu partout dans l'archipel, sous des formes différentes et avec d'autres composantes, mais débouchant le plus souvent sur la reconstitution d'une structure forestière au sein du terroir agricole ${ }^{6}$. Dans les conditions actuelles de réduction du couvert forestier et de perte de contrôle des ressources par les populations autochtones, ces agroforêts sont souvent les derniers garants de la permanence de la ressource forestière dans son ensemble, aussi bien au niveau du paysage qu'au niveau des représentations et des usages. Mais elles constituent aussi la base du succès des systèmes de production agricole qui les englobent.

L'agroforêt constitue un modèle de gestion de ressources naturelles renouvelables, mais aussi un paradigme nouveau pour le développement durable des zones forestières. Au-delà des enseignements qu'elle apporte en matière de sylviculture et de conservation, l'agroforêt montre surtout comment la gestion globale des ressources forestières pourrait évoluer dans un sens totalement original en s'intégrant davantage à l'agriculture sans pour autant épouser les modèles de plantation monospécifique.

En réponse aux besoins en termes de reforestation liés à la régression souvent irréversible des forêts naturelles sous les tropiques, la foresterie classique ne s'est développée que dans une direction : la mise au point de techniques d'établissement et de gestion de plantations forestières industrielles, ces nouveaux faciès de forêt totalement artificialisés qui n'ont de "forestier" que le bois. L'agroforêt montre qu'il existe d'autres solutions techniques à ce problème de reconstitution des ressources et des fonctions forestières. Sur son modèle, on peut aisément envisager des écosystèmes moins complexes qu'une forêt primaire, mais écologiquement plus proches d'elle que les plantations industrielles.

L'agroforêt est au centre d'un processus de transformation du milieu naturel destiné à la production dans lequel la pratique agricole a joué et joue encore un rôle catalytique essentiel'. La structure biologique recréée prend la forme de la forêt qu'elle remplace, mais elle reste, au niveau de sa gestion et de sa perception par les paysans, intimement intégrée au système de production agricole, dont elle représente certainement l'élément majeur. L'agroforêt est conçue et gérée comme une entreprise agricole com-
5. Economistes et sociologues ont clairement montré que cette perception négative ( $\mathrm{C} f$. la tradégie des communaux de Hardin, 1968) résultait d'une confusion entre propriété commune et accès libre (Berkes et al., 1989; Aguilera-Klink, 1994): cette confusion, qui s'avère particulièrement redoutable en ce qui concerne les ressources forestières en Indonésie (Tjondronegoro, 1987), parait rester savamment entretenue par certains groupes: ne permet-elle pas d'affirmer l'incapacité des populations locales à gérer sans les dilapider les ressources communes, et par voie de conséquence de nier les droits d'usage de ces populations ?

6. Voir entre autres les publications sur les agroforèts à rotins de Kalimantan : Weinstock, 1985 ; les agroforèts à hévéa de Sumatra : Colfer et al. 1988, Gouyon et al. 1993 ; les agroforêts à hévéa de Kalimantan : Dove 1993, de Jong 1994 ; les agroforêts à tembawang de Kalimantan : Momberg, 1993 ; Sundawati , 1993 ; les agroforêts à fruitiers de Kalimantan : Salafsky, 1994.

7. Les surfaces occupées par les agroforêts sur la seule ile de Sumatra sont estimées à plus de 3,5 millions d'hectares (de Foresta et Michon, 1993). 
merciale. Ses résultats économiques sont bien meilleurs que ceux que l'on peut espérer tirer d'une forêt où l'on exploiterait les mêmes ressources, et restent les meilleurs garants de sa reproduction, fondée sur des bases écologiques - la restauration de structures biologiques diversifiées quasiment autonomes.

Par rapport aux plantations classiques, l'agroforêt permet aussi une conservation importante de la diversité biologique, corollaire d'une certaine diversité d'utilisation. Cette diversité biologique - espèces et relations biologiques entre espèces - est essentielle dans la mesure où elle maintient ouvertes d'autres possibilités d'utilisation et d'évolution. La transformation agroforestière ne constitue pas une spécialisation irréversible, ni du milieu, ni des structures productives, biologiques ou économiques, ni même des fonctions sociales qui lui sont associées, et ceci est un phénomène unique dans les exemples d'intensification agricole de par le monde.

L'agroforêt dans sa totalité représente un patrimoine (Mary, 1986), l'appropriation de la terre remplaçant la possession traditionnelle des arbres. Cependant, le capital foncier d'une agroforêt s'enrichit d'une structure productive - les arbres planifiée sur le long terme conférant en quelque sorte une valeur ajoutée au patrimoine et justifie a posteriori le droit de regard du lignage sur sa gestion. Dans l'établissement d'une agroforêt, le retour sur investissement est tel qu'il constitue une garantie supplémentaire de pérennité. Et les arbres, signe de cet investissement, sont de fait valorisés au même titre que la terre. Dans l'agroforêt, on ne compte pas en hectares, mais le plus souvent en nombre d'arbres productifs. Les restrictions d'usage concernent aussi bien la terre - prohibition de vente - que les arbres - interdiction de couper les bons producteurs. On retrouve ici, au sein de structures d'appropriation agricoles, des logiques plus caractéristiques des gestions forestières. Cette prise en compte de logiques forestières constitue une révolution certaine pour l'agriculture, où le long terme n'atteint que rarement la dizaine d'années.

La qualité essentielle des agroforêts réside avant tout dans les effets synergiques de cette intégration entre le monde forestier et la pratique agricole, et c'est sans doute là l'apport essentiel du paradigme agroforestier défini ci-dessus à la problématique du développement durable. Accentuer les côtés agricoles au dépens des aspects forestiers, ou vanter telle ou telle qualité forestière de l'agroforêt peut cependant avoir une importance réelle dans le contexte particulier de la définition de politiques locales de développement. Là où les forestiers rechignent à reconnaitre les droits des populations locales sur les ressources forestières, il vaudra sans doute mieux pousser à la reconnaissance du caractère "agricole" des agroforêts.

Enfin, il convient de souligner l'intérêt scientifique des agroforêts, qui ouvrent de nouvelles possibilités de dialogue, non seulement entre forestiers et agronomes, mais surtout entre spécialistes des sciences humaines et biologistes.

\section{CONCLUSION : VERS UNE RÉAPPROPRIATION DES RESSOURCES FORESTIĖRES PAR LES COMMUNAUTÉS LOCALES ?}

La disparition des forêts tropicales représente souvent plus un problème social et idéologique qu'un problème technique. $\grave{A}$ la lecture des vingt dernières années de l'histoire des forêts en Indonésie, l'exploitation forestière apparaît, avec l'accord tacite du gouvernement indonésien et des diverses organisations internationales ayant soutenu et parfois orienté la politique officielle, comme le fer de lance d'un processus de conversion plus ou moins contrôlé des terres forestières (Durand, 1994). Nombre de forêts exploitées pour le bois ont été irréversiblement transformées. À travers la "nécessaire" mise en valeur des terres une fois déforestées, cette conversion permet de dicter aux "îles extérieures" une philosophie très javanaise du développement agricole qui privilégie nettement l'agriculture inten- sive et spécialisée. Elle permet également d'effacer du même coup les revendications possibles des populations locales sur les ressources.

Dans ce contexte de dépossession organisée, qui se retrouve souvent sous les tropiques où la terre est avant tout "fores. tière” et propriété de l'État, l'intérêt des agroforêts, qui se situent en dehors du cadre conceptuel et administratif des forêts naturelles, devient évident. Parce qu'elle n'est pas une forêt naturelle mais bien une forêt reconstituée, parce qu'elle peut ainsi se réclamer du monde agricole bien que ne correspondant pas au modèle classique des plantations, l'agroforêt peut permettre aux paysans de réaffirmer leur ancienne souveraineté sur les ressources naturelles. La stratégie agroforestière, surtout, ouvre un champ totalement nouveau pour les négociations entre forestiers, responsables du développement agricole, et communautés locales sur la question de la gestion durable des ressources naturelles. Elle peut permettre de définir de nouvelles bases légales, reconnues et agréées par toutes les parties prenantes, pour l'appropriation, l'utilisation et la gestion des terres forestières déjà transformées. Elle peut aussi proposer des modèles aussi bien techniques que juridiques et sociaux pour une gestion durable des ressources forestières menacées par l'exploitation actuelle, et garantir ainsi la permanence non seulement de ressources, mais aussi de forêts et de sociétés originales.

(Article reçu le 13 janvier 1995).

\section{Références}

Aguilera-Klink F. (1994). Some notes on the misuse of classic writings in economics on the subject of common property Ecological Economics, 9, 221-228.

Anderson A.B. éd. (1990). Alternatives to deforestation. Steps towards sustainable use of the Amazon rainforest, New York. Columbia University Press.

Alexandre D.-Y. (1989). L'arbre et le maintien des potentialités agricoles en zone intertropicale humide. In Eldin M., Milleville P., eds., Le risque en agriculture, Paris, Orstom, 115-152.

Berkes F. éd. (1989). Common property resources : ecology and community-based sustainable development, London, Belhaven Press.

Berkes F., Feeny D., McKay B.J., Acheson J.M., (1989). The benefits of the commons, Nature, 340 .

Colfer C.J.P., Gill D.W., Fahmuddin A. (1988). An indigenous agri- 
cultural model from West-Sumatra : a source of scientific insight, Agricultural systems, 26, 191-209.

Dove M. R. (1985). The Agroecological mythology of the Javanese and the political economy of Indonesia, Indonesia, 39 Dove M. R. (1993). Smallholder rubber and swidden agriculture in Borneo : a sustainable adaptation to the ecology and economy of the tropical forest, Economic Botany, 47 (2), 136-147. Dunn F. L. (1975). Rain-forest collectors and traders: a study of resource utilization in modern and ancient Malaya, Kuala Lumpur.

Dupain D. (1994). Une règion traditionnellement agroforestière en mutation : le Pesisir, rapport Orstom-Biotrop-Cnearc-Eitarc, Montpellier, France.

Durand F. (1994), Les forêts en Asie du Sud-Est. Recul et exploitation. Le cas de I'Indonésie, Paris, L'Harmattan.

Foresta H. de, Michon G. (1991). Agroforesteries indonésiennes ; systèmes et approches, Communication à l'Atelier "Quelles agroforestries pour l'Orstom ", Paris, Orstom.

Foresta H. de, Michon G. (1992). Complex agroforestry systems and conservation of biological diversity 2/ For a larger use of tradition agroforestry trees as timber in Indonesia, a link between environmental conservation and economic development. In Yap Son-kheong, Lee Su-win, éd., In harmony with nature. International conference on the conservation of tropical biodiversity, Kuala Lumpur, Malaysia, The Malayan Nature Journal (Golden jubilee issue), 45 (1), 488-500.

Gillis M. (1988). Public policies and the misuse of forest resources, Cambridge University Press.

Gouyon A., de Foresta H., Levang P. (1993). Does Jungle Rubber deserve its name? An analysis of rubber agroforestry systems in Southeast Sumatra, Agroforestry Systems, 22, 181. 206.

Hardin G. (1968). The tragedy of the commons, Science, 162 1243-1248.

Jong W. de (1994). Communication at the "International symposium on management of tropical forests in Southeast Asia", Oslo, Mars 1994.

Levang P. (1989). Systemes de production et revenus familiaux In Pain M., éd., Transmigration et migrations spontanées en Indonésie, Jakarta-Paris, Departement Transmigrasi- Orstom, 193-283.

Levang P. (1992). Pahmungan, Penengahan, Balai Kencana. Enquête agro-économique dans la région de Krui (Lampung), Rapport de mission Soft-Orstom-Biotrop.

Mary F. (1986). Agroforêts et sociètes. Etude comparée de trois systèmes agroforestiers indonésiens, thèse de docteur-ingénieur, Ensa-Montpellier.

Mary F., Michon G. (1987). When agroforests drive back natural forests : a socio-economic analysis of a rice/agroforest system in South-Sumatra, Agroforestry Systems, 5, 27-55.

McKay B, Acheson J.M. (1987). The question of the commons: the culture and ecology of communal resources, Tucson, University of Arizona Press.

Michon G. (1985). De l'homme de la forét au paysan de l'arbre agroforesteries indonesiennes, these de doctorat, U.S.T.L., Montpellier.

Michon G. (1993). Gestion des ressources naturelles : la voie agroforestière indonésienne, Arbres, Forêts et Communautés rurales, 5, 28-36.

Michon G., Foresta H. de (1994). Forest resource management and biodiversity conservation : the indonesian agroforest model. Communication à l'Atelier UICN u Biological diversity conservation outside protected areas : overview of traditional agroecosystems n, Madrid, mai 1994.

Momberg F. (1993). Indigenous knowledge systems. Potentials for social forestry development : resource management of Land-Dayaks in West-Kalimantan, Berlin, Technische Universität Berlin.

Nair P.K.R. (1989). Agroforestry systems in the tropics, Dordrecht, Kluwer Academic Publisher and Icraf.
Peluso N. L. (1992 a). The ironwood problem : (mis) management and development of an extractive rainforest product Conservation Biology, 6 (2), 210-219.

Peluso N. L. (1992 b). Rich forest, poor people. Resource control and resistance in Java, Berkeley, University of California Press. Rappard F. W. (1937), Oorspronkelijke bijdragen : de damar van Bengkoelen (The damar of Bengkulu). Tectona, 1 (30), 897-915. Redford K. H., Padoch C. (1989). Conservation of neotropical Columbia University Press.

Salafsky N. (1994). The forest garden project : an ecological and economic study of a locally developed land-use system in West. Kalimantan, Indonesia, Ph.D. Thesis, Duke University, Durham, North Carolina, USA.

Schlager E., Ostrom E. (1992). Property-rights regimes and natural resources : a conceptual analysis, Land Economics, 68 (3), 249-262.

Sibuea T.T.H., Herdimansyan D. (1993). The variety of mammal forests. Working from traditional resource use, New York,

species in the agroforest areas of Krui (Lampung), Muara Bungo (Jambi), and Maninjau (West-Sumatra), rapport ORSTOM-BIOTROP/HIMBIO (UNPAD), Bandung, Indonesia.

Siebert S. F. (1989). The dilemma of dwindling resource : rat$\tan$ in Kerinci, Sumatra, Principes, 32 (2), 79-97.

Sundawato L. (1993). The Dayak garden systems in Sanggau district, West-Kalimantan. An agroforestry model, MSc. thesis, Faculty of Forestry, Georg-August University, Göttingen. Thiollay J. M. (1994). Are traditional agroforests an alternative for the conservation of rainforest bird diversity ? Three case studies in Sumatra. To be published in Conservation Biology.

Tjondronegoro S. M. P. (1987). Notes on tenure and agroforestery in Indonesia. In J. B. Raintree, éd., Land, trees and tenure, Nairobi and Madison, Icraf and the Land tenure center, 253-259.

Weinstock J.A. (1983). Rattan : ecological balance in a Borneo rainforest swidden, Economic botany, 37 (1), 58-68.

\section{ABSTRACT : Peasant agroforestry strategies and sustainable development : the damar agroforests of Sumatra (Indonesia)}

In Indonesia, for the last twenty years, the increasing number of conflicts between the State and local communities about the use and the control of resources leads to the accelerated dilapidation of forest resources. This wasting goes along with a rapid degradation of the environment and the issue of sustainable development of forest areas is at the very heart of the national debate. The reexist nevertheless positive examples of indigenous management systems of forest resources, especially in agroforestry.

Between plantation and forest, Indonesian agroforests represent an original strategy for the appropriation and use of natural resources by peasant communities, combining both agricultural goals and forest reconstruction.

Via the history and the analysis of an agroforest in Sumatra, this paper addresses the contribution of the "agroforest" concept to the debate on the dynamics and the use of renewable resources in the framework of sustainable development. Putting the stress on the biological mechanisms and the social strategies which allowed, more than the conservation of a particular forest resource, the restoration of the forest as a resource in its totality, we discuss the viability of this agroforestry strategy in a context rather unfavorable to the control of forest resources by peasants. 